\title{
Myelofibrosis and Antiphospholipid Syndrome Presenting With Adrenal Insufficiency Due to Bilateral Adrenal Hemorrhage: A Case Series
}

\author{
Helena Robbins ${ }^{\mathrm{a}}$, Amira Hegab ${ }^{\mathrm{b}}$, Helen Ward ${ }^{\mathrm{c}}$, Dipesh C. Patel ${ }^{\mathrm{b}}$, Anne Miller ${ }^{\mathrm{d}}$, Allan Irvine ${ }^{\mathrm{e}}$, \\ Gerard S. Conway ${ }^{b}$, Thang S. Han ${ }^{c, f}$
}

\begin{abstract}
Adrenal hemorrhage is rare but appears to occur more frequently in patients with underlying hematological conditions. We report two recent patients with underlying hematological disorders who presented with adrenal insufficiency due to bilateral adrenal hemorrhage. The first case is a 59-year-old woman known to have myelofibrosis. This patient was being treated with warfarin for left leg deep vein thrombosis which developed after splenectomy. She was admitted to hospital with vomiting and abdominal pain. The second case is an 84-year-old male patient with antiphospholipid syndrome and diabetes mellitus who was treated with warfarin and insulin. He was admitted with sudden onset of nausea, hypotension and recurrent hypoglycemia despite cessation of insulin therapy. In both cases, bilateral enlarged adrenal glands consistent with hemorrhage were detected resulting in adrenal insufficiency. It is important that Hematologists and Endocrinologists alike should recognise symptoms of adrenal insufficiency in patients with hematological disorders, particularly those being treated with antiplatelet medi-
\end{abstract}

Manuscript accepted for publication July 29, 2011

${ }^{\text {a } D e p a r t m e n t ~ o f ~ G a s t r o e n t e r o l o g y, ~ A s h f o r d ~ a n d ~ S t ~ P e t e r ' s ~ N H S ~}$ Foundation Trust, UK

${ }^{\mathrm{b}}$ Department of Endocrinology, University College London Hospitals NHS Foundation Trust, UK

${ }^{\mathrm{c}}$ Department of Endocrinology, Ashford and St Peter's NHS Foundation Trust, UK

${ }^{\mathrm{d} D e p a r t m e n t ~ o f ~ H a e m a t o l o g y, ~ A s h f o r d ~ a n d ~ S t ~ P e t e r ' s ~ N H S ~ F o u n d a t i o n ~}$ Trust, UK

eDepartment of Radiology, Ashford and St Peter's NHS Foundation Trust, UK

${ }^{\mathrm{f}}$ Corresponding author: Thang S Han, Department of Endocrinology, St Peter's Hospital, Guildford Road, Chertsey, KT16 0PZ, UK.

Email: t.s.han@doctors.org.uk

doi: $10.4021 / \mathrm{jem} 31 \mathrm{w}$ cations or anticoagulants.

Keywords: Hematological disorders; Anticoagulants; Addisonian crisis

\section{Introduction}

Patients with hematological disorders such as antiphospholipid syndrome (APS) and primary myelofibrosis are susceptible to thromboembolic complications. These patients often require thromboprophylaxis such as antiplatelet medications and, if thrombosis has occurred, coumarin [1]. In these patients, adrenal hemorrhage resulting in adrenal insufficiency is a rare but serious complication, with subtle and variable clinical presentations. Here, we report two patients with underlying hematological disorders who both presented with adrenal hemorrhage leading to adrenal insufficiency.

\section{Case Report}

\section{Case 1}

A 59-year-old lady with a diagnosis of essential thrombocythemia in 1991 which had transformed to myelofibrosis in 2009 (negative for JAK2 $2^{\mathrm{V} 617 \mathrm{~F}}$ mutation) had been treated with aspirin, hydroxycarbamide, danazol and recombinant erythropoietin. In September 2010 she underwent splenectomy for increasing transfusion requirements. Post-operatively, she developed a left leg deep vein thrombosis and was treated with warfarin.

Four weeks later, she was admitted with vomiting and left upper quadrant abdominal pain for 24 hours. These symptoms were associated with weakness, general lethargy, fevers (peak temperature $38.5^{\circ} \mathrm{C}$ ) and dyspnoea. On examination, there was no change in skin pigmentation or rash. She was tachypneic but not hypoxic. Her supine blood pressure was 145/75 mmHg. Arterial blood gases were obtained showing respiratory alkalosis. Admission urea and electrolytes were 

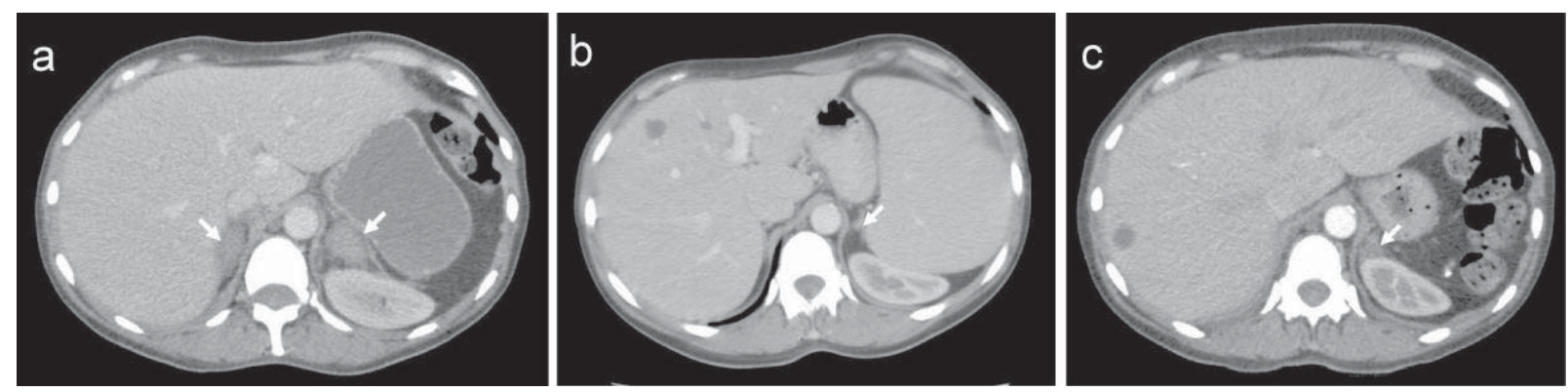

Figure 1. CT scan of the abdomen of a 59-year-old female patient with myelofibrosis showing evidence of enlarged bilateral adrenal hemorrhage (arrows) (a). CT scan of the abdomen of the same patient four months previously showing splenomegaly and normal left adrenal (arrow), the right adrenal is barely visible between the liver and right diaphragmatic crus (b), and 7 months after admission showing reduction in the size of both adrenal glands (only the left adrenal gland as indicated by arrow is visible in this image) compared to the previous examination (Fig. 1a) consistent with resolving hemorrhage (c).

normal, levels of alkaline phosphatase, aspartate transaminase and lactate dehydrogenase were elevated. C-reactive protein (CRP) was mildly elevated, but creatinine kinase, troponin I, amylase and glucose levels were all normal.

Her hemoglobin $(\mathrm{Hb})$ and white cell count (WCC) were within reference limits and platelets were $458 \times 10^{9} / 1$. The international ratio (INR) was 2.3 and had never been above the treatment range before admission. Computerised tomography (CT) scan of her abdomen revealed acutely enlarged bilateral adrenal hemorrhage (Fig. 1a). The adrenal architecture was shown to be entirely normal 15 weeks previously (Fig. 1b).

A random cortisol was abnormally low $(57 \mathrm{nmol} / \mathrm{l})$ and adrenal insufficiency was confirmed by short Synacthen test (SST) (peak cortisol levels of $87 \mathrm{nmol} / \mathrm{l}$ ). Her adrenal androgens were undetectable. Hydrocortisone was administered immediately at $50 \mathrm{mg}$ IM qds leading to rapid improvement in symptoms and was switched to $20 \mathrm{mg}+10 \mathrm{mg}+10 \mathrm{mg}$ PO regimen. Once well, the dose of hydrocortisone was reduced to a physiological dose of $10 \mathrm{mg}+5 \mathrm{mg}+5 \mathrm{mg}$ PO regimen and fludrocortisone of $100 \mu \mathrm{g}$ PO daily added. Meanwhile, warfarin was replaced with low molecular weight heparin injections. Blood cultures, performed on three separate occasions, were all negative. Bone marrow biopsy was performed and cytogenetic study showed an abnormal clone and loss of part of chromosome 7 consistent with myeloid disorder. Ziehl-Neelsen stain of bone marrow and T-SPOT ${ }^{\circledR}$ TB test both showed no evidence of tuberculosis bacilli.

Repeat CT scans of the adrenals were performed four weeks (not shown) and seven months (Fig. 1c) after admission showing progressive decrease in size and resolution of hemorrhage. Repeat SST eight weeks after the patient's admission showed persistent adrenal insufficiency (peak cortisol levels of $119 \mathrm{nmol} / \mathrm{l})$.

\section{Case 2}

An 84-year-old man known to have APS with recurrent pul- monary emboli, ulcerative colitis and type 2 diabetes mellitus was treated with Warfarin and Novomix-30 90 units per day. He was admitted in late 2010 with hyperosmolar hyperglycemic syndrome and severe right leg cellulitis requiring 72 hours of intravenous antibiotics. The warfarin dose was increased from the usual dose of $2-3 \mathrm{mg}$ to $5 \mathrm{mg}$ due to a sub-therapeutic INR of 2.1 (target INR 3 - 4).

He was readmitted 48 hours later with sudden onset of back pain and nausea. He was hypotensive and had evidence of recurrent hypoglycemia despite cessation of insulin therapy. The admission INR was 6.9. His urea and electrolytes were mildly deranged. The levels of CRP were elevated (148 $\mathrm{mg} / \mathrm{l})$. His full blood counts were within reference limits. Suspected acute adrenal insufficiency was confirmed by SST (peak cortisol of $132 \mathrm{nmol} / \mathrm{l}$ ). A CT scan of the abdomen demonstrated evidence of bilateral hemorrhage (Fig. 2). Hydrocortisone of $100 \mathrm{mg}$ IV was administered acutely followed by a $20 \mathrm{mg}+10 \mathrm{mg}+10 \mathrm{mg}$ PO regimen, leading to a rapid clinical improvement. He was discharged 10 days after admission on fludrocortisone $50 \mu \mathrm{g}$ daily and physiological

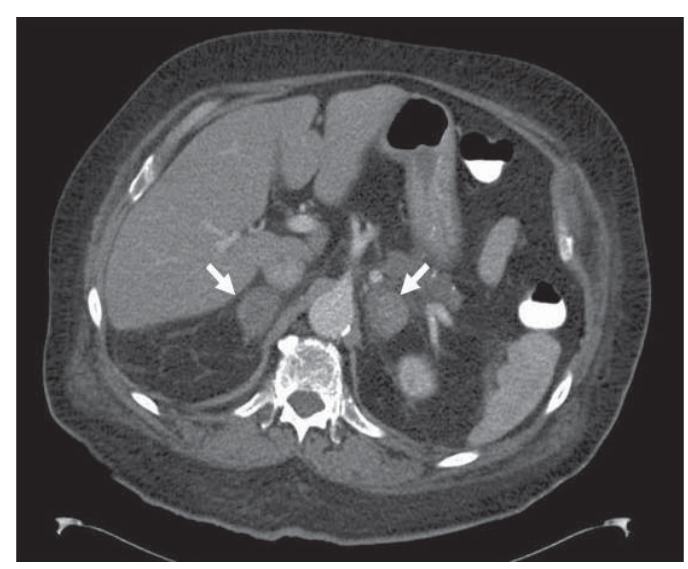

Figure 2. CT scan of the abdomen of an 84-year-old male patient with APS showing evidence of bilateral adrenal hemorrhage. 


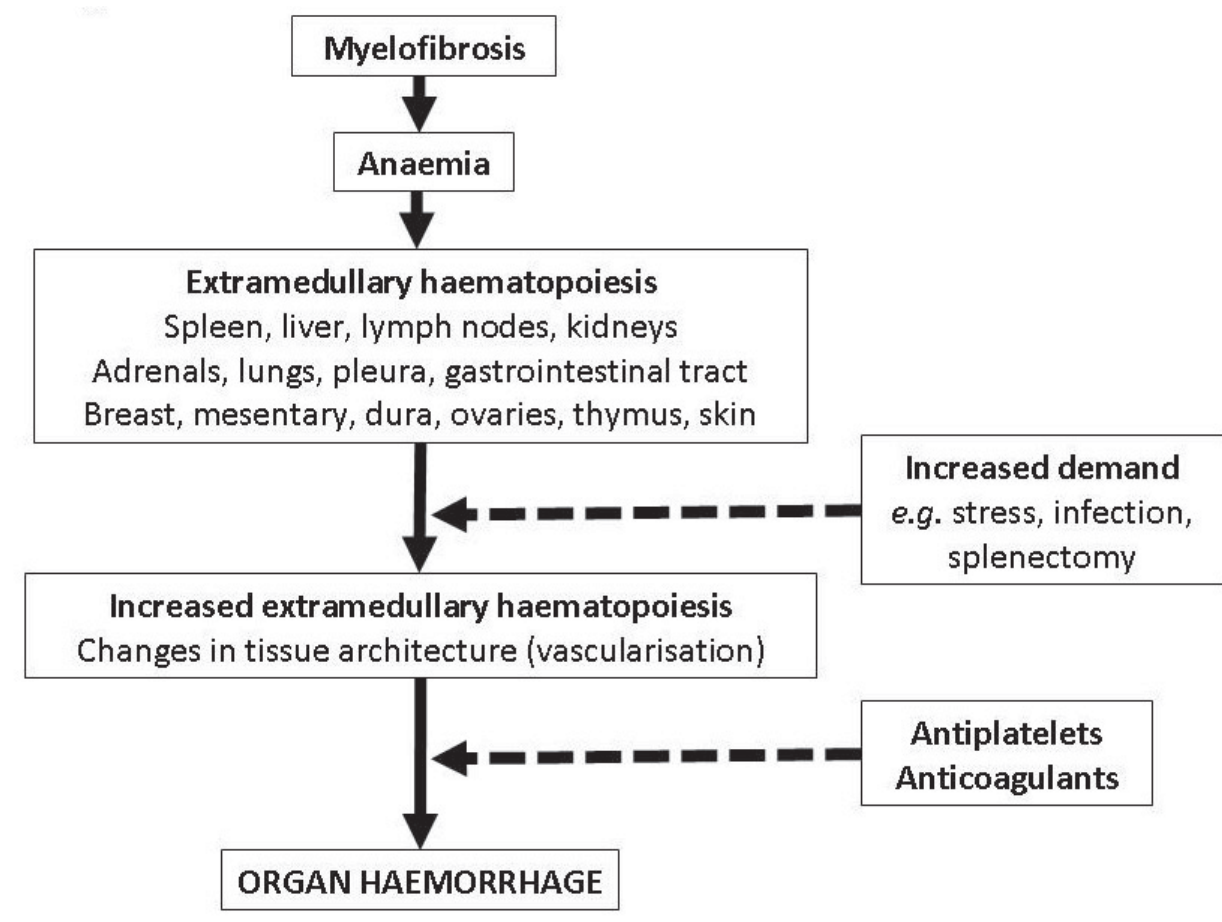

Figure 3. Possible mechanisms leading to organ hemorrhage in patients with myelofibrosis treated with antiplatelet or anticoagulant medications (dashed lines indicate potentiation effect).

dosing of hydrocortisone. Follow-up assessment of adrenocortical function is planned.

\section{Discussion}

We report two patients with underlying hematological disorders who were admitted recently with bilateral adrenal hemorrhage and associated adrenal insufficiency. This lifethreatening complication is not uncommonly reported in patients with APS $[2,3]$ but rarely reported in those with myeloproliferative disorders such as myelofibrosis [4], polycythemia vera [5] or essential thrombocythemia [5, 6]. To the best of knowledge, no previous reports have demonstrated CT images capturing normal adrenals prior to dramatic bilateral hemorrhagic enlargement within a short period. The underlying etiological mechanisms remain uncertain. In the case of myelofibrosis, possible explanation includes infiltration of abnormal stem cells, thrombosis followed by hemorrhagic necrosis or adrenal extramedullary hematopoiesis (infra vida). In the case of APS, thrombosis followed by hemorrhage has been postulated. About $6 \%$ of patients with myeloproliferative disorders present with hemorrhage at diagnosis (mostly gastrointestinal). The risk of bleeding increases in the elderly and with aspirin use. It has been suggested that acquired type 2 von Willebrand disease may occur in these patients and can contribute to hemorrhage, but only in those with extreme thrombocytosis [7]. It is unlikely that antiplatelet or anticoagulant treatment alone could cause bilateral adrenal hemorrhage since the majority of patients treated with these agents for other conditions do not commonly develop adrenal hemorrhage. This complication may also occur with other conditions including meningococcal disease (Waterhouse-Friderichsen syndrome), tuberculosis and histoplasmosis.

It is plausible that when extramedullary hematopoiesis develops in an organ, tissue microarchitectural changes and angioneogenesis occur, rendering that organ at greater risk of hemorrhage. We postulate that in patients with myeloproliferative disorders, adrenal hemorrhage occurs as a result of changes in tissue architecture due to adrenal extramedullary hematopoiesis which are potentiated by antiplatelet medications, or anticoagulants (Fig. 3). In a previous report of a patient with myelofibrosis who presented with adrenal hemorrhage, fine needle aspiration showed adrenal extramedullary hematopoiesis and autopsy revealed a mixture of extramedullary hematopoiesis and hemorrhage [4]. In patients with myelofibrosis, extramedullary hematopoiesis may occur in many organs including the adrenal glands [7]. Increased cutaneous extramedullary hematopoiesis after splenectomy in a patient with myelofibrosis has been observed [8]. Interestingly, since our patient underwent splenectomy only three months prior to adrenal hemorrhage, it is possible that, as a compensatory response to the loss of splenic hematopoiesis and the addition of high demand related to stress or anemia, the adrenals could have developed extramedullary hemato- 
poiesis, thus predisposing them to hemorrhage from anticoagulation.

Only a handful of patients have been reported to have shown recovery of adrenal function. For example, a 30-yearold woman with APS presented with bilateral adrenal hemorrhage, after three months the adrenal morphology was normal on CT scan and after 7 months, her adrenal function recovered completely as demonstrated by SST [9]. In another report, adrenal hemorrhage induced by coumarin (post-operative therapy for pelvic fractures) in a 35-year-old man completely recovered after 9 months [10]. At the time of this report, our 'Case 1' underwent a SST after two months showing a marginal improvement in response to Synacthen but remained insufficient.

\section{Conclusion}

Physicians should be aware of the potential for adrenal insufficiency secondary to adrenal hemorrhage in patients with hematological disorders being treated with antiplatelet medications or anticoagulants.

\section{Consent}

Written informed consent was obtained from both patients for publication of this case report and any accompanying images.

\section{Authors' Contributions}

All authors were involved in study design, drafting the manuscript and revising it critically and read and approved the final manuscript.

\section{Competing Interests}

The authors declare that they have no competing interests.

\section{References}

1. Tuthill JI, Khamashta MA. Management of antiphospholipid syndrome. J Autoimmun. 2009;33(2):92-98.

2. Arnason JA, Graziano FM. Adrenal insufficiency in the antiphospholipid antibody syndrome. Semin Arthritis Rheum. 1995;25(2):109-116.

3. Goldenberg A, Tollin SR (1998). Massive bilateral adrenal hemorrhage and adrenal crisis in two patients with the primary antiphospholipid syndrome. Endocrinologist, 8:365-368.

4. King BF, Kopecky KK, Baker MK, Clark SA. Extramedullary hematopoiesis in the adrenal glands: CT characteristics. J Comput Assist Tomogr. 1987;11(2):342-343.

5. Michiels JJ, Berneman Z, Schroyens W, Krestin GP. Bilateral adrenal swelling as a cause of chest, back, and upper abdominal pain in essential thrombocythemia and polycythemia vera is due to microvascular ischemic thrombosis rather than to hemorrhage. Ann Hematol. 2002;81(12):691-694.

6. Kojima K, Fujii N, Takata S, Tezen T, Morikawa $\mathrm{T}$, Kaneda K, Takenaka K, et al. Bilateral adrenal hemorrhage in essential thrombocythemia. Ann Hematol. 2002;81(6):350-351.

7. Steensma DP, Richard RE (2007) Myeloproliferative disorders. American Society of Hematology SelfAssessment Program. 2007 (1): 172-227.

8. Hocking WG, Lazar GS, Lipsett JA, Busuttil RW. Cutaneous extramedullary hematopoiesis following splenectomy for idiopathic myelofibrosis. Am J Med. 1984;76(5):956-958.

9. Vengrove MA, Amoroso A. Reversible adrenal insufficiency after adrenal hemorrhage. Ann Intern Med. 1993;119(5):439.

10. Clarke BL, Beyer HS, Weisman I, Sharp B, Nippoldt TB (1995) Rapid recovery from adrenal insufficiency due to bilateral adrenal hemorrhage. Endocrinologist, 5:312315. 